\title{
Prélude: enquête autour d' un tapuscrit retrouvé
}

\author{
Elara Bertho
}

L'enquête a commencé par hasard. Lorsque je cherchais les sources africaines de l'historien Yves Person, j' eus la surprise de trouver non pas seulement des cahiers de notes de ses "sources orales" qui ont fait sa renommée, mais également plusieurs sources écrites africaines. Cette découverte dans les archives de son ancienne université - à la Bibliothèque de Recherches Africaines de Paris 1 , rue Malher ${ }^{1}$ - est étonnante à plus d'un titre. D' abord, il existait dans les années 1950 plusieurs récits écrits africains de l'histoire de Samori Touré, et nulle part Yves Person ne souligne l'importance de cette littérature endogène rédigée par des "lettrés locaux."2 En outre, l'une d'entre elles se détache du lot: il s'agit du texte de 110 pages d'un certain Djiguiba Camara, intitulé "Essai d'histoire locale." C' est ce texte que nous éditons ici. Il raconte (comme son titre l'indique) l'histoire de sa région, autour du petit village de Damaro en Haute Guinée, sur les contreforts des monts Simandougou. Il dépeint les migrations mythiques des Camara, les guerres de Samori Touré, les guerres de conquête coloniale, jusqu'à aboutir à l' époque de la rédaction du document, en 1955. J'étais convaincue que cette source était intéressante mais j' étais bien en peine de reconstituer les circonstances de production d'un tel texte. Le patronyme de l'auteur, extrêmement commun en Guinée, rendait l'identification des descendants délicate. Un sondage aux archives d'Aix-en-Provence ne donnait rien de très concluant sur cet auteur.

Travaillant de conserve avec Marie Rodet, je commence à annoter le texte pour le rendre compréhensible en vue d'une édition. L'argumentation est en effet parfois obscure et nécessite d'être explicitée. J'entame une relecture systématique de Samori Touré, une révolution dioula d'Yves Person pour mettre

1 L'œuvre de référence d'Yves Person, qui l'a occupé toute sa vie, est la somme Samori, une révolution dyula (Person 1968-1975). Archives personnelles "Yves Person," BRA (Bibliothèque de Recherches Africaines), Paris 1. J' ai assisté Michèle Raffutin, l' archiviste et la directrice de la bibliothèque, dans le premier état des lieux de ce fonds et dans l' entreprise de classement et de référencement sur le site Calames. À l'époque, les archives d'Yves Person étaient sur des étagères non verrouillées, ce qui les rendaient disponibles au tout venant. Désormais, elles sont consultables sur demande et entièrement référencées. Le catalogue est en ligne sur le site Calames: http://www.calames.abes.fr/pub/\#details?id=FileId-1255, consulté le 24 mai 2018.

2 Je retranscris deux de ces sources écrites africaines en annexe de ma thèse: Bertho 2016: 6o9635 (“Entretiens” de Babou Kondé et “Notes historiques” de Tidiane Dem). 
en avant les passages du tapuscrit qui ont servi à l'historien français. Je me rends compte à cette occasion que Djiguiba Camara est certainement l'une des sources africaines les plus régulièrement citées dans des épisodes stratégiques de la conquête coloniale. Il fournit également de précieux renseignements sur la période précoloniale, notamment pour les premières années du règne de Samori Touré au moment de son expansion en Haute Guinée. Ce patient travail philologique, certes ingrat mais permettant néanmoins de prouver plusieurs de nos intuitions sur l'usage des sources africaines en regard des sources européennes, s' arrête brutalement à la réception d' un email.

Le 30 juin 2015, El Hadj Daouda Damaro Camara et le Commandant N'Faly Camara cosignent un courrier nous intimant de cesser tout travail d' édition et nous menaçant de procès si nous "subtilisons même une ligne de ce manuscrit," nous rappelant à cette occasion que "les lois guinéennes et françaises protègent les œuvres et leurs auteurs." Le ton et l' agressivité de la lettre nous laissent un moment sans voix, Marie et moi. Nous répondons bien sûr en nous excusant, sentant bien que nous avions touché des susceptibilités que nous ne pouvions deviner.

Il semblait que le document ait eu une vie postérieure à l'usage qu' en avait fait Yves Person, puisque El Hadj Daouda Damaro Camara, le fils de Djiguiba, affirmait qu'il avait abondamment retravaillé le texte de son père. Cela, nous ne le savions pas, et c'était la première mention du devenir de cet étrange manuscrit qu' est "Essai d'histoire locale." Les réponses abruptes des descendants à la suite de cet email nous dissuadent de continuer plus avant notre travail d'édition et d'annotation. Je continue ma thèse consacrée à Samori Touré; les mois passent. Nous nous faisons à l'idée que cette édition ne se fera pas.

En décembre 2015, par un autre jeu de hasard, le député Mohamed Touré, fils de Sékou Touré l' ancien président de la Guinée, rencontre Marie Rodet et promet de jouer les intermédiaires avec la famille Camara. Dans l'échiquier politique de la Guinée contemporaine, il est à l' exact opposé de la famille Camara, dont l'un de ses membres est l'éminent Ahmadou Damaro Camara, que l'on appelle "l' Honorable" comme il se doit au pays, et qui n' est rien de moins que le président de la majorité présidentielle à l'Assemblée Nationale et le conseiller spécial du Président Alpha Condé. Pourtant, malgré ces différents politiques, Mohamed Touré intervient en notre faveur, et l'Honorable est particulièrement sensible à cette intercession peu orthodoxe: nous sommes officiellement invitées à rencontrer la famille Camara à Conakry.

Grâce à un financement de la British Academy, nous arrivons, sans trop savoir à quoi nous attendre, à Conakry en décembre 2016. Nous sommes reçues avec curiosité par plusieurs représentants de la famille Camara. Ceux issus directement du village de Damaro accolent ce nom à leur patronyme - ce 
même Damaro qui était présent dans le document "Essai d'histoire locale." Lors d'une visite de courtoisie à l'Honorable, nous apprenons que nous sommes attendues au village, à 800 kilomètres d'ici. Les voitures nécessaires à notre déplacement sont déjà réservées, El Hadj Daouda Damaro Camara viendra avec nous, ainsi que plusieurs autres membres de la famille qui souhaitent se joindre à l'équipée. Puisque tout semble décidé, nous nous plions au programme qui a été établi pour nous et, après deux jours pleins de voyage, nous arrivons enfin au village. Nous sommes surprises de constater que la concession de Djiguiba Camara a été repeinte pour l'occasion: en lettres bleues et rouges se détachent, éclatantes, son nom et la durée de son mandat à la tête de la chefferie du canton. Nous sommes venues avec une caméra pour enregistrer les entretiens: elle servira autant à filmer les réceptions organisées pour célébrer notre venue que les témoignages émouvants de ceux qui ont connu Djiguiba Camara. Mawa Koné, l'une de ses anciennes femmes, pleure de joie à notre arrivée. Il semble que la caméra soit un gage de notre engagement dans la mise en valeur du patrimoine culturel du village. Contrairement à ce que je redoutais, elle sert à délier les langues et les souvenirs. L'on retrouve pour nous deux vieilles machines à écrire, sorties de larges cantines en fer. Dans les étagères de la concession se côtoient des papiers jaunis du temps de l'administration françaises, des lampes tempêtes et des affiches électorales. Des généalogies, des listes d'habitants des villages environnants, des collections du Bulletin de l'Afrique française sont entassés pêle-mêle. El Hadj Daouda Damaro Camara, qui était dans la même voiture que nous tout au long du trajet, nous accorde sa confiance petit à petit et nous raconte l'histoire de son père.

Djiguiba Camara avait écrit "Essai d' histoire locale" avec pour ambition de le faire publier. El Hadj Daouda Damaro Camara accuse Yves Person de lui avoir soutiré son œuvre en échange d'une promesse fallacieuse de publication en France. Il accuse par ailleurs l'historien français d'avoir plagié son père. ${ }^{3}$ Toute sa vie, El Hadj Daouda Damaro Camara a eu à cœur de compléter le document de son père, reprenant de nombreuses fois, pour nous expliquer cette entreprise encyclopédique ${ }^{4}$ la métaphore de la construction: son père

3 Il ne s'agit pas dans cette édition de porter des accusations contre l'une ou l'autre des parties: présentant les dessous de la recherche africaniste, nous nous attachons surtout à mettre en lumière le statut d'un savant guinéen méconnu, d' analyser son rapport à un historien et administrateur français (en l'occurrence, Yves Person), tout en décrivant le souvenir douloureux pour la famille Camara de cet "échange" intellectuel. Les deux versants du souvenir et de l'écriture de l' histoire sont ici considérés.

4 Sur la notion de "tentation encyclopédique," voir la thèse de Chavoz 2018. 
a jeté les fondations d'une maison, tandis que lui s'est chargé du reste du chantier, embellissant les murs, ajoutant des carreaux aux fenêtres, finissant la toiture. Aujourd'hui, son texte compte plus de deux mille pages et cette œuvre immense est tout à la fois composite et fascinante: elle englobe l' histoire de la traite transatlantique, l'histoire des mythes de fondation des villes alentours, l'histoire de la colonisation, elle intègre aussi des listes de patronymes malinké, des listes de proverbes... Toujours à l' occasion de notre venue, El Hadj Daouda Damaro Camara trouve les financements pour faire imprimer et relier son œuvre gigantesque. C'est la première fois qu' il l'emmène au village et les deux volumes élégamment reliés trôneront au milieu de la concession tout au long de notre séjour à Damaro. Petits et grands s' approprient le texte, en lisent quelques lignes, parcourent des yeux les nombreuses illustrations, commentent à plusieurs certains passages.

Au retour à Conakry, un conseil de famille se réunit pendant que nous récupérons des fatigues du trajet. El Hadj Daouda et Mamadi Damaro Camara viennent nous retrouver à l' issue de la réunion, dont on mentionne avec euphémisme qu' elle fut "un peu longue." La famille nous donne solennellement le droit d'éditer en Europe le manuscrit de leur aïeul, et nous demande de tout faire pour promouvoir son œuvre. ${ }^{5}$ El Hadj Daouda et Mamadi Damaro Camara sont chargés de suivre pour le compte de la famille élargie l' avancée des opérations. Il est important de mentionner ici que Djiguiba Camara avait seize femmes: Daouda est, de ses fils, celui qui est chargé de l'héritage spirituel du père parce qu'il connaît le mieux ses textes et l'ensemble de son travail de collecte des récits de la région, et Mamadi Damaro Camara est le plus âgé des petits-enfants. À eux deux, ils représentent les intérêts de la famille Damaro Camara. Cet accord nous permet de penser à nouveau au chantier de publication, en laissant présager des pistes d' analyses multiples que nous n' aurions jamais imaginées avant cet étrange voyage de terrain.

Non seulement Djiguiba Camara avait rencontré Yves Person et avait été l'une de ses meilleures sources africaines, mais surtout il se voulait historien, et la publication devait confirmer ce statut de "lettré." Il a tout mis en œuvre pour accéder à cette légitimité symbolique, qui lui a été refusée de son vivant et dont la famille garde un souvenir amer. Notre propre entreprise d'édition, que nous pensions être une mise en valeur des intermédiaires coloniaux, ravivait en réalité des peurs postcoloniales et nous venions reproduire, soixante-dix ans

5 Suite à l'accord conclu avec la famille Camara concernant de la présente publication, Jan Jansen s' est également engagé à aider la famille Camara à publier la volumineuse version retravaillée par El Hadj Daouda Damaro Camara, en collaboration avec le African Studies Center Leiden, Pays-Bas. 
après le passage d'Yves Person, une douloureuse expérience ressentie comme un pillage. L'on n' est pas chercheuses blanches en Afrique impunément.

Plus formidable encore, peut-être, ce texte de Djiguiba Camara a eu une vie tout à fait romanesque après le passage d'Yves Person: il a été repris par son fils Daouda, il fut emmené en Côte d' Ivoire lorsque celui-ci décida de fuir le régime de Sékou Touré en 1972, il fut amplifié patiemment pendant tout ce temps jusqu'à aujourd' hui, et il est revenu avec lui en Guinée. Depuis la fin des années 1960, El Hadj Daouda Damaro Camara reprend même contact avec Yves Person pour retrouver des photographies de son père et solliciter de nouveau un soutien à la publication; ${ }^{6}$ il entretient également une correspondance avec Denise Bouche, Ibrahima Baba Kaké, Ahmadou Hampaté Bâ. ${ }^{7}$ En un mot: ce texte est l' œuvre de sa vie.

Ce dialogue commencé véritablement à l'occasion de notre venue n'a depuis jamais cessé. Nous communiquons régulièrement par mails ou par téléphone. Je suis revenue à Conakry puis à Damaro, avec Jan Jansen, en janvier et février 2018, grâce à une subvention britannique. ${ }^{8}$ Puis de nouveau rapidement en avril 2018. À chaque fois, El Hadj Daouda Damaro Camara nous accompagne et nous raconte avec un enthousiasme toujours égal le parcours de son père, mille anecdotes sur la période coloniale et le souvenir de ses réalisations dans la région.

6 Lettre de Yves Person à Daouda Camara, datée de mars 1968, Université de Dakar, Faculté des Lettres et Sciences Humaines, département d'histoire. Lettre de Yves Person à Daouda Camara, datée de 14 avril 1972, Université de Paris, Faculté des Lettres et Sciences Humaines, Centre de recherches africaines. Archives personnelles de El Hadj Daouda Damaro Camara, Conakry.

7 Entretien avec El Hadj Daouda Damaro Camara, 23 décembre 2016, Conakry.

8 ESRC "Resilience in West African Frontier Communities" (ES/Roo280o/1) dirigé par Marie Rodet et mené en collaboration avec Friederike Lüpke, Bakary Camara et Elara Bertho et l'oNG malienne Donkosira. 


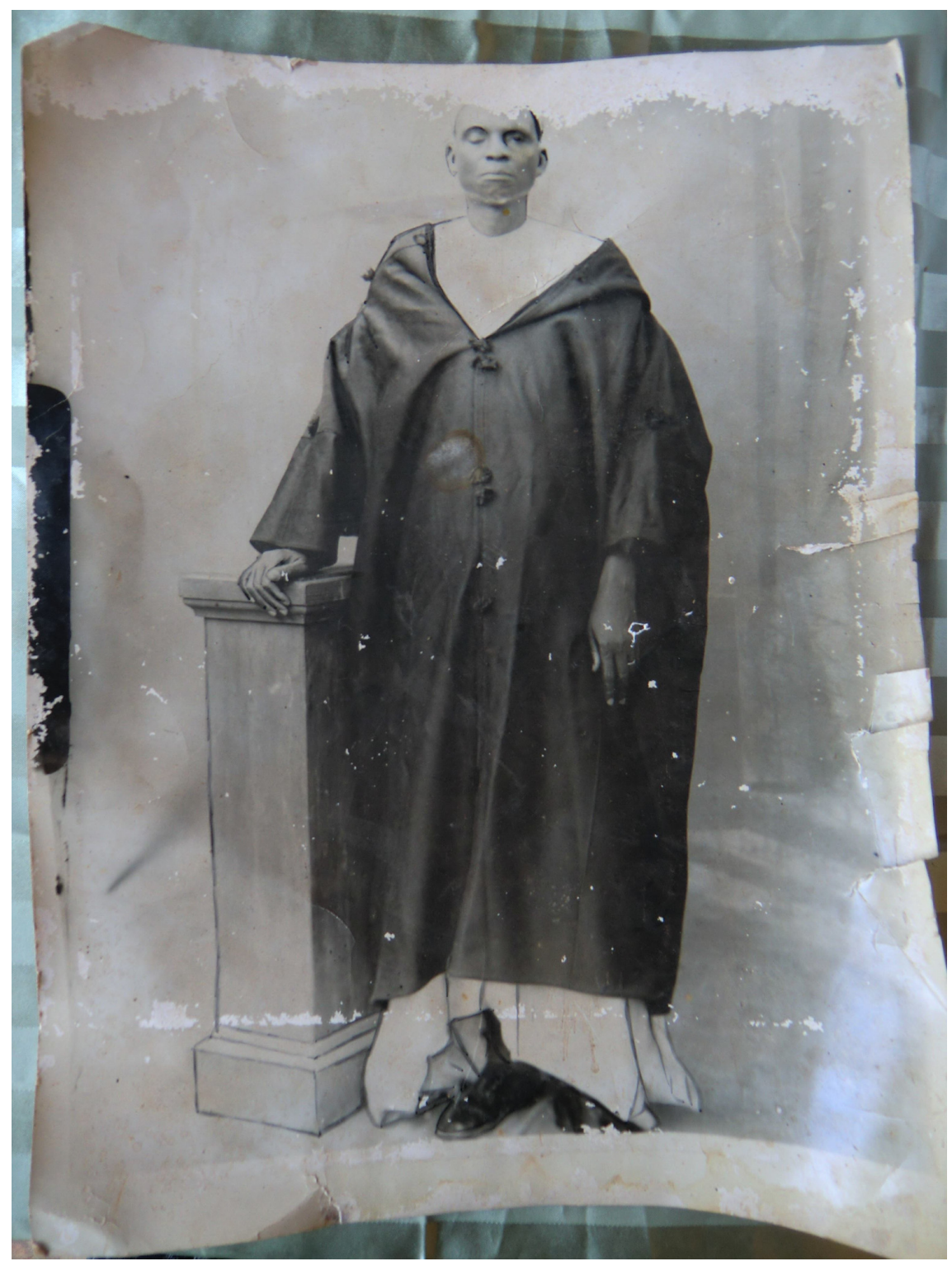

ILL. 2

Djiguiba Camara, photographie familiale conservée à Damaro / Djiguiba Camara, family photo kept in Damaro 